\title{
Sistematização da assistência de enfermagem em uma gestante com Ruptura Prematura das Membranas Ovulares (RPMO): um relato de experiência
}

\author{
Systematization of the assistance of nursing in uma gestante with Premature Rupture of \\ Membranes Ovulares (PRMO): an experience story
}
Sistematización de la ayuda del cuidado en uma gestante con la Ruptura Prematura de las Membranas Ovulares (RPMO): una historia de la experiencia

José Carlos da Luz Gonçalves ${ }^{1 *}$, Paula Abitbol Lima ${ }^{1}$, Kelberthy Kayo da Silva Balieiro ${ }^{1}$, Francilene Moraes Pereira ${ }^{1}$, Luci Selma Ferreira de Freitas Farias ${ }^{1}$, Jessica de Souza Pereira ${ }^{1}$, Ruthlene Freitas Gonçalves ${ }^{1}$, Tyago Andrade de Lima' ${ }^{1}$, Jaqueline Cardoso Marcena².

\section{RESUMO}

Objetivo: Relatar a experiência vivenciada, por acadêmicos do 9o período do curso de Bacharelado em Enfermagem do Centro Universitário Metropolitano da Amazônia (UNIFAMAZ), ao elaborar o Processo de Enfermagem a uma gestante com Ruptura Prematura das Membranas Ovulares (RPMO). Detalhamento do Caso: Gestante L.C.S.V, 30 anos, no $3^{\circ}$ trimestre de gestação (34 semanas e 1 dia) no $9^{\circ}$ dia de internação hospitalar com quadro de RPMO. Trata-se de um estudo descritivo, de abordagem qualitativa, do tipo relato de experiência que se deu pelo acompanhamento e evolução diária da gestante. Discussão: Durante o estágio supervisionado em obstetrícia, os discentes tiveram a oportunidade de realizar a aplicabilidade eficaz da Sistematização da Assistência de Enfermagem, que resultou na melhora do quadro clínico da paciente, potencializando o cuidado oferecido pela equipe de enfermagem. Considerações Finais: Por meio deste estudo, fica evidente a realização da anamnese e exame físico criterioso para subsidiar na elaboração dos diagnósticos de enfermagem e suas intervenções.

Palavras-chave: Cuidados de enfermagem, Trabalho de parto prematuro, ROPREMA.

\begin{abstract}
Objective: To tell the lived deeply experience, for academics of $9^{\circ}$ period of the course of Bacharelado in Nursing of the Metropolitan University Center of the Amazônia (UNIFAMAZ), when elaborating the Process of Nursing to a gestante with Premature Rupture of Membranas Ovulares (RPMO). Detailing of the Case: Gestante L.C.S.V, 30 years, in 3o trimester of gestation (34 weeks and 1 day) in 9o day of hospital internment with RPMO picture. One is to a descriptive study, qualitative boarding, the type experience story that if gave for the accompaniment and daily evolution of the gestante. Quarrel: During the period of training supervised in obstetricses, the learning had had the chance to carry through the efficient applicability of the Systematization of the Assistance of Nursing, that resulted in the improvement of the clinical picture of the patient, being potencializando the care offered for the nursing team. Considerações Final: By means of this study, it is evident the accomplishment of anamnese and criterioso physical examination to subsidize in the elaboration of the nursing disgnostic and its interventions.
\end{abstract}

Keyword: Cares of nursing, Work of premature childbirth, ROPREMA.

${ }^{1}$ Centro Universitário Metropolitano da Amazônia (UNIFAMAZ), Belém-PA. *E-mail: josecarlos-luz@outlook.com

${ }^{2}$ Universidade do Estado do Pará (UEPA), Belém-PA.

SUBMETIDO EM: 1/2019

ACEITO EM: 2/2019

PUBLICADO EM: 4/2019

REAS/EJCH | Vol. Sup. 22 | e282 | DOI: https://doi.org/10.25248/reas.e282.2019 Página 1 de 5 


\section{RESUMEN}

Objetivo: Para decir profundamente vivida la experiencia, para el académico del período 9o del curso de Bacharelado en el oficio de enfermera del centro metropolitana de la universidad del Amazônia (UNIFAMAZ), al elaborar el proceso del cuidado a un gestante con la ruptura prematura de Membranas Ovulares (RPMO). El detallar del caso: Gestante L.C.S.V, 30 años, en el trimestre $3^{\circ}$ de la gestación (34 semanas y 1 día) en el día 9º de la internación del hospital con el cuadro de RPMO. Uno está a un estudio descriptivo, el subir cualitativo, el tipo historia de la experiencia que si dio para el acompañamiento y la evolución diaria del gestante. Pelea: Durante el período del entrenamiento supervisado en obstetricses, el aprender había tenido la ocasión de llevar con la aplicabilidad eficiente de la sistematización de la ayuda del oficio de enfermera, que dio lugar a la mejora del cuadro clínico del paciente, siendo potencializando que el cuidado ofreció para el equipo del oficio de enfermera. Final de Considerações: Por medio de este estudio, es evidente la realización de la examinación física anamnese y del criterioso a subvencionar en la elaboración del oficio de enfermera disgnostic y de sus intervenciones.

Palabra-clave: Cuidados del oficio de enfermera, Trabajo del parto prematuro, ROPREMA.

\section{INTRODUÇÃO}

A Ruptura Prematura das Membranas Ovulares (RPMO) consiste em uma complicação obstétrica caracterizada pela ruptura espontânea do âmnio e do cório antes do início do trabalho de parto em grávidas com idade gestacional acima de 20 semanas, aumentando o risco para parto prematuro, sofrimento fetal e infecções ascendentes (PIATO S, 2009).

A Ruptura Prematura das Membranas Ovulares (RPMO) pode ocorrer a termo em $8 \%$ das gestações tendo como principal consequência as infecções intrauterinas que aumenta com o tempo de ruptura e é geralmente seguida pelo início rápido do parto dentro de 24 horas, assim como, a RPMO Pré-termo $(<37$ semanas), onde mesmo na vigência de tratamento expectante, na maioria dos casos a gestante entra em trabalho de parto no prazo de uma semana ocorrendo em $3 \%$ das gestações e a RPM Pré-viável $(<23$ semanas) onde, incide em $1 \%$ das gestações e inúmeras condições estão associadas à compressões dos pulmões fetais pela oligoidramnia e levam a hipoplasia pulmonar (MONTENEGRO CAB e FILHO JR, 2015).

Existem diferentes fatores responsáveis pela debilitação das membranas ovulares, tais como: Infecções bacterianas que ao se multiplicarem produzem uma substância denominada fosfolipase, onde desempenha um papel mais relevante na gênese da ruptura. $O$ tabagismo, embora o mecanismo pelo qual os derivados do fumo atuam no sentido de diminuir a resistência das membranas ovulares ainda não se encontra estabelecido, e a deficiência de Colágeno, nessa situação a fragilidade das membranas é intrínseca e não se deve á atuação dos fatores responsáveis pela anomalia (PIATO S, 2009).

A ruptura prematura das membranas ovulares tem sido responsável por aproximadamente $1 / 3$ dos casos de parto prematuro, portanto, essa complicação obstétrica requer profissionais de enfermagem capacitados para desenvolver o Processo de Enfermagem (PE) de forma eficaz, garantindo uma assistência adequada ao paciente (MONTENEGRO CAB e FILHO JR, 2015).

Para isso, surge então a Sistematização da Assistência de Enfermagem (SAE), cujo objetivo é organizar o trabalho profissional quanto ao método, pessoal e instrumentos, tornando possível a operalização do Processo de Enfermagem (PE). A SAE consiste em uma metodologia científica que vem sendo cada vez mais implantada na pratica assistencial, garantindo maior segurança aos pacientes, melhora na qualidade da assistência e maior autonomia aos profissionais por meio do Processo de Enfermagem (PE) que é definido como a execução da SAE em toda a sua prática (HERMIDA PMV e ARAÚJO IEM, 2006).

Segundo a resolução do COFEN 358/2009, a utilização desse instrumento se organiza em cinco etapas: coleta de dados (histórico), diagnóstico de enfermagem, planejamento de enfermagem dos resultados esperados, implementação da assistência de enfermagem e a avaliação de enfermagem, que garante ao professional a qualificação do gerenciamento do cuidado e o planejamento de suas atividades, além de servir como guia para suas ações (SILVA JP, et al., 2015).

Estudos desenvolvidos atualmente indicam diferentes dificuldades por meio dos enfermeiros na implantação da Sistematização da Assistência de Enfermagem (SAE), entre elas: a falta de conhecimento

REAS/EJCH | Vol. Sup. 22 | e282 | DOI: https://doi.org/10.25248/reas.e282.2019 Página 2 de $\mathbf{5}$ 
teórico e metodológico dos modelos assistenciais, deficiência na discussão da temática ainda em sua graduação e a grande influência do modelo biomédico/cartesiano (MEDEIROS AL, et al., 2013).

Desta forma, este estudo tem como objetivo relatar a experiência vivenciada, por acadêmicos do 9o período do curso de Bacharelado em Enfermagem do Centro Universitário Metropolitano da Amazônia (UNIFAMAZ), ao elaborar o Processo de Enfermagem a uma gestante com quadro de Ruptura Prematura das Membranas Ovulares.

\section{DETALHAMENTO DO CASO}

Foi realizado um estudo descritivo, de abordagem qualitativa, do tipo relato de experiência que se deu pelo acompanhamento e evolução diária de uma gestante com quadro de Ruptura Prematura das Membranas Ovulares (RPMO).

O estágio supervisionado, da disciplina "Obstetrícia" do $9^{\circ}$ período do curso de Bacharelado em Enfermagem do Centro Universitário Metropolitano da Amazônia (UNIFAMAZ), ocorreu em uma unidade Materno Infantil situada na cidade de Belém-PA, a qual atende $100 \%$ o Sistema Único de Saúde (SUS) e é cadastrada como referência na atenção á gestante de alto risco e ao recém-nascido, no período de 23/08/2018 a 12/09/2018.

Os estágios curriculares, vem proporcionar para os discentes um senso crítico e a capacidade de elaboração da melhor assistência de enfermagem a gestante que esteja hospitaliza por um quadro clínico de alto risco, apoiada em bases cientificas, metodológicas, éticas e legais, considerando as necessidades humanas básicas.

Os dados coletados para a realização da SAE, ocorreram durante a prática através de uma busca ativa do prontuário, anamnese e exame físico criterioso da gestante, assim como a interação com os familiares presentes e da observação. A partir dos dados obtidos, tornou-se possível identificar os diagnósticos de enfermagem com o apoio da Classificação de Diagnóstico de Enfermagem da North American Nursing Diagnosis Association Internacional (NANDA).

\section{DISCUSSÃO}

Durante o estágio supervisionado em obstetrícia, os discentes tiveram a oportunidade de realizar a aplicabilidade eficaz da Sistematização da Assistência de Enfermagem, que em dado momento se deu pelo acompanhamento da paciente do estudo L.C.S.V., sexo feminino, 30 anos, no $3^{\circ}$ trimestre de gestação (34 semanas e $1 \mathrm{dia}$ ), natural e procedente de Tomé-Açú/Pa, no $9^{\circ} \mathrm{DIH}$, e portadora da Hipertensão Arterial Sistêmica (HAS).

A hipertensão crônica na gestação é definida como a elevação da pressão arterial (PA>140 x 90 $\mathrm{mmHg}$ ), observada antes da concepção ou de 20 semanas da gestação (NHBPEP, 2000). Sibai B (2002) vem ressaltar que a mensuração da pressão arterial deve ocorrer em ao menos duas ocasiões com intervalo mínimo de 4 horas entre elas.

De acordo com NHBPEP (2000), o diagnóstico de hipertensão crônica torna-se bastante simples, porém, o que dificulta esse diagnóstico precoce está associado ao início tardio do pré-natal (>20 semanas), após a ocorrência da queda fisiológica da pressão no $2^{\underline{a}}$ trimestre e pela inconstância da história pré-concepcional da gestante.

$\mathrm{Na}$ anamnese, L.C.S.V. apresentava boa aceitação e adaptação do quadro clínico e ao ambiente hospitalar, porém, manifestou alterações emocionais no decorrer de sua internação como tristeza, ansiedade, preocupação, medo e nervosismo pela falta de informação sobre seu prognóstico, recebendo apoio familiar e da equipe multidisciplinar.

Como forma de tranquiliza-la, os discentes buscaram proporcionar um ambiente agradável no qual favoreceu um diálogo claro e objetivo entre a equipe e a paciente que esclareceu suas dúvidas e queixas 
relatada neste momento, onde minimizou seu sofrimento diante do quadro vivenciado. Ao término, os acadêmicos elaboraram um plano assistencial de acordo com a evolução e diagnósticos de enfermagem traçados pela Classificação de Diagnóstico de Enfermagem da NANDA Internacional.

\section{Evolução de Enfermagem}

As 14:00h foi realizada visita de enfermagem a gestante L.C.S.V., cujo histórico obstétrico é (5 $5^{a}$ gestação, 2 partos e 2 abortos anteriores. Data da Última Menstruação (DUM): 25/12/2017, Data Prevista do Parto (DPP): 01/10/2018, Idade Gestacional (IG) pela Ultrassonografia (USG): 35 semanas e 3 dias / Idade Gestacional (IG) de acordo com a Data da Última Menstruação (DUM): 34 semanas e 3 dias), encontra-se em trabalho de parto relacionado com o quadro de Rotura Prematura das Membranas Ovulares, consciente, orientada, ansiosa, com medo, colaborativa, eupneica, acianótica, anictérica com pele e mucosas íntegras e normocoradas. Queixa principal: Dor em baixo ventre, cefaleia, perda de tampão mucoso e de líquido amniótico. Sinais Vitais: Normocárdica, normotensa, eupneica e afebril. Ao Exame Físico: Couro cabeludo íntegro e limpo, pele e mucosas hipocoradas, sem presença de gânglios infartados. Tórax: simétrico com boa expansividade, ausculta cardíaca (AC): bulhas cardíacas normofonéticas em 2 Tempos e sem sopros, ausculta pulmonar (AP): murmúrios vesiculares presentes e sem ruídos adventícios, mamas: turgidas e simétricas, mamilos protusos com presença de colostro espontâneo. Abdômen gravídico com movimentos fetais presentes, batimentos cardíacos fetais (BCF): 145 batimentos por minuto (bpm), altura uterina 30 centímetros, feto único em situação longitudinal, posição dorso a direita, apresentação cefálica. Genitália íntegra apresentando perda de tampão mucoso com sangue. Membros Superiores e Inferiores (MMSS/II): simétricos, apresentando cacifo (-), com boa perfusão periférica, Acesso Venoso Periférico (AVP) salinizado em antebraço esquerdo. Aceita bem a dieta oferecida. Padrão de eliminação urinário alterado, caracterizado pelo aumento de idas ao banheiro em curtos espaços de tempo e padrão de eliminação intestinal preservado e espontâneo.

ID: Ruptura Prematura das Membranas Ovulares. Condutas: Orientações sobre resultados de exames, vacinas, esclarecimentos de dúvidas. Aplicada escala numérica de dor que deu como resultado 3 , classificando como dor leve em baixo ventre devido contrações uterinas que alivia ao se deitar em decúbito lateral esquerdo melhorando o retorno venoso pela descompressão da veia cava inferior.

\section{Sistematização da Assistência de Enfermagem (SAE)}

De acordo com os dados obtidos na evolução e apoio do NANDA, os autores traçaram os seguintes diagnósticos de enfermagem (DE): Risco de infecção intrauterina relacionada com a ruptura da barreira amniótica; Ansiedade caracterizado por relato verbal e relacionada com a gravidez e seu desfecho; Dor aguda caracterizado por expressão facial e corporal relacionada com as contrações uterinas; Risco de infecção relacionado ao acesso venoso periférico; Medo caracterizado por relato verbal, relacionado a possibilidade se sofrimento ou morte fetal, assim como, trocas de gases fetal prejudicada caracterizada pela diminuição do líquido amniótico relacionado a RPMO.

A partir dos diagnósticos de enfermagem definidos em concordância com o NANDA, os cuidados oferecidos que serão apresentados. Ressaltamos que os mesmos foram estabelecidos levando em consideração a história da paciente e suas principais queixas e complicações.

As principais intervenções identificadas para o risco de infecção intrauterina são: Atentar para a presença de líquido amniótico e secreção vaginal e anotar suas características, quantidade e odor. Verificar batimentos cárdicos fetais (BCF) atentando para bradicardia ou taquicardia a cada $4 / 4 \mathrm{~h}$. As intervenções nos casos de ansiedade e do medo. Cabe a enfermagem e a equipe multidisciplinar fornecer informações de maneira clara e direta sobre o quadro clinico da paciente e seus possíveis desfechos, permitindo que a paciente faça perguntas e retire suas dúvidas. Atentar para alterações fisiológicas associadas a ansiedade como: elevação da pressão arterial sistêmica, taquicardia ou taquipneia mãos frias e úmidas.

Para Schiavo RA et al. (2018), a ansiedade na gestação é uma das características comuns de alterações psicológicas durante a gravidez, que pode sofrer variação em sua intensidade quando se considera os diferentes aspectos socioculturais em que a gestante está inserida.

Schetter CD e Tanner $L$ (2012) afirmam que, quando as gestantes começam apresentar taxas elevadas de ansiedade, podem ressaltar em complicações e/ou intercorrências obstétricas, como parto/nascimento pré-maturo e de baixo peso. 
Para o diagnóstico de dor as intervenções consistem em: Aplicar escala numérica de dor, e anotar sua frequência, localização e intensidade a cada $6 / 6$ h e se necessário. Proporcionar medidas de conforto como: troca de decúbito, massagenterapia e técnicas de relaxamento a cada $6 / 6 \mathrm{~h}$ ou conforme necessidade.

Quanto ao risco de infecção relacionado ao acesso venoso central, as intervenções consistem em: Avaliar o local de acesso intravenoso quanto aos sinais de flebite ou infiltração de $6 / 6 \mathrm{~h}$. Trocar curativo do acesso diariamente ou se necessário, assim como, trocar acesso venoso periférico a cada $92 \mathrm{~h}$.

As intervenções em casos de troca de gases fetais prejudicada relacionada a Roprema são: Estimular a ingesta hídrica materna (manhã/tarde/noite). Atentar para soroterapia rigorosa conforme prescrição médica. Verificar batimentos cárdicos fetais (BCF) atentando para bradicardia ou taquicardia a cada $4 / 4 \mathrm{~h}$.

Diante das intervenções de enfermagem realizadas nos diagnósticos acima, os discentes tiveram a oportunidade de vivenciar com a paciente L.C.S.V., uma evolução significativa e satisfatória do seu quadro clínico. Essa experiência se deu a partir da aplicabilidade eficaz da Sistematização da Assistência de Enfermagem potencializando os cuidados oferecidos pelos enfermeiros, técnicos e/ou auxiliar de enfermagem que lidam diretamente com o paciente.

Sendo assim, o processo de enfermagem realizado pelos discentes contribuiu para o amadurecimento pessoal e profissional durante a graduação, apontando para a relevância do enfermeiro (a) na assistência a gestante de alto risco.

\section{CONSIDERAÇÕES FINAIS}

Por meio desse estudo, observou-se que a pratica humanizada contribuiu para diminuir o sofrimento vivenciado pela paciente durante sua internação hospitalar. Desta forma, fica evidenciado a importância de o enfermeiro (a) assistir o paciente de forma integral através da utilização da SAE, uma vez que a sistematização da sua assistência é uma ferramenta imprescindível que facilita o seu cuidado prestado.

\section{REFERÊNCIAS}

1. PIATO S. Complicações em Obstetrícia. Barueri, SP. Manole, 2009.

2. MEDEIROS AL, et al. Desvelando Dificuldades Operacionais da Sistematização da Assistência de Enfermagem na Perspectiva da Grounded Theory. Rev. Eletr. Enf. 2013 jan/mar;15(1):44-53

3. MONTENEGRO CAB, FILHO JR. Rezende Obstetrícia Fundamental. 13 ed. Rio de Janeiro: Guanabara Koogan, 2015.

4. NATIONAL HIGH BLOOD PRESSURE EDUCATION PROGRAM WORKING GROUP REPORT ON HIGH BLOOD PRESSURE IN PREGNANCY. Am J Obstet Gynecol 2000; 163. 1991.

5. HERMIDA PMV, ARAÚJO IEM. Sistematização da Assistência de Enfermagem: Subsídios para Implantação. Rev Bras Enferm 2006 set-out, 59(5): 675-9.

6. SCHETTER CD, TANNER L. Anxiety, depression and stress in pregnancy: Implications for mother, children, research, and practice. Current Opinion in Psychiatry, 2012, 25(2), 141-148.

7. SIBAI B. Chronic Hypertension in Prograncy. Obstet gynecol. 2002. 100: 369.

8. SCHIAVO RA, et al. Variáveis Associadas á Ansiedade Gestacional em Primigestas e Multigestas. Trends Psychol., Ribeirão Preto, vol. 26, oㅜ 4, p. 2091--2104 - Dez/2018.

9. SILVA JP, et al. Sistematização da Assistência de Enfermagem na Graduação: Um Olhar Sob o Pensamento Complexo. Rev Latino-Am 2015 jan-fev, 23(1): 59-66.

10. NORTH AMERICAN NURSING DIAGNOSIS ASSOCIATION. Diagnósticos de Enfermagem da NANDA: definições e classificação 2009-2011. Porto Alegre: Artmed, 2009.606p.

11. ZUGAIB M. Protocolos assistenciais: clínicas obstétricas FMUSP. 3ª ed. São Paulo, Atheneu, 2017.

12. MELSON KA, et al. Enfermagem materno-infantil: planos de cuidados. Editora Reichmann \& Affonso. $3^{\text {a }}$ ed. Rio de Janeiro, 2002. 